\title{
Property instantiation in conceptual combination
}

\author{
EDWARD J. WISNIEWSKI \\ University of North Carolina, Greensboro, North Carolina
}

\begin{abstract}
In four experiments, I examined how a property in one concept is transferred to a second concept during conceptual combination. The results suggest that people instantiate properties: that is, they use a specific representation of a property in the modifier concept to construct a new version of that property that is specific to the combination. If people are instantiating properties, then the modifier property should match its counterpart in the combination to the extent that the modifier and head noun are similar. This observation leads to a variety of predictions (supported by the experiments) about interpretations of similar and dissimilar combinations and about plausibility, preference, and similarity judgments associated with such interpretations. The results argue against an alternative view of transfer that posits that, in general, abstract representations of properties are copied from one concept to another. In this paper, I describe various processing accounts of instantiation and discuss the implications of the instantiation view for theories of metaphor, conceptual combination, and induction.
\end{abstract}

Many cognitive phenomena involve the use of knowledge of a domain or concept to understand another. Analogies are one such phenomenon. For example, a child learning about the atom may understand the analogy "the atom is like the solar system" by using knowledge that the planets revolve around the sun in the solar system to infer that electrons revolve around the nucleus of the atom. As a second case, understanding noun-noun combinations involves using knowledge of the modifier concept to understand the meaning of the combination. For example, understanding kangaroo squirrel involves the use of knowledge in the modifier noun kangaroo (e.g., knowledge that kangaroos hop) to infer that a kangaroo squirrel is a squirrel that hops. Categorization is a third phenomenon that can be viewed in this way. In categorizing a relatively long, slithering thing making a rattling sound as a rattlesnake, one may use knowledge about rattlesnakes in general to infer that this particular rattlesnake is poisonous and could bite you.

A pervasive metaphor in cognitive psychology is that the use of knowledge in one domain to understand another involves "copying" that knowledge from one domain to the other. This view is explicit in many models of analogy and metaphor in which an important component of understanding involves the transfer of knowledge from the source domain to the target domain by a process called copy and substitution (Falkenhainer, Forbus, \& Gentner, 1989; Gentner, 1983, 1989; Holyoak \& Thagard,

The author thanks Douglas Medin for very helpful discussions of this work. Thanks also to Kristen Carpenter, Tasneem Sheikl, and especially Merigan Kline for help in running the experiments and analyzing the data. Bob Dylan provided some of the inspiration for this work. Correspondence should be addressed to E. J. Wisniewski, Department of Psychology, University of North Carolina, P.O. Box 26164, Greensboro, NC 27402-6164 (e-mail: edw@uncg.edu).

—Accepted by previous associate editor Brian H. Ross
1989; Hummel \& Holyoak, 1997). In this case, a predicate of the source domain is copied to the target domain, and the arguments to the predicate are replaced with appropriate arguments of the target domain. For example, understanding the analogy "the hydrogen atom is like our solar system" includes copying the predicate revolves from solar system to atom and substituting the arguments (planets, sun) in the solar system domain with corresponding arguments (electrons, nucleus) in the atom domain (Gentner, 1983, p. 159).

The copy metaphor is also implicit in recent models of induction (Heit \& Rubinstein, 1994; Osherson, Smith, Wilkie, Lopez, \& Shafir, 1990; Sloman, 1993). These approaches account for a variety of psychological phenomena involving the degree to which people believe that a property is true of a conclusion category given that it is true of one or more premise categories. In doing so, they assume that the representation of the property is the same in the premise and conclusion categories. For example, the feature-based induction model (Sloman, 1993) learns an association between properties of the premise category and a new property that transfers to the conclusion category to the degree that the conclusion category shares the premise category's properties. Thus, the identical property of the premise category is now linked to the conclusion category (achieving the same effect as copying the property from the premise to the conclusion category).

Despite the pervasiveness of the copy metaphor, intuition suggests that knowledge transfer may not always be this straightforward. For example, consider the novel combination zebra clam interpreted as "a clam with stripes." In this case, people use the property stripes of the modifier concept zebra to understand zebra clam. However, it seems implausible that people understand zebra clam by transferring a literal copy of the specific stripes of a zebra to clam. Instead, the stripes of a zebra clam seem different from those of a zebra. Although they might show an alternating dark and light pattern, as they do in 
a zebra, they might also be shorter and thinner and closer together than those of a zebra (to be consistent with the size of a clam). In fact, there exists a mollusk called a zebra mussel, which has this pattern of stripes.

More generally, Wisniewski (1997) suggested that people employ an interactive construction process in combining such concepts as zebra and clam. According to this view, a property of the modifier noun acts as a source for the creation, or "instantiation," of a new version of that property in the head noun of the combination. Furthermore, both concepts provide constraints on how the new version is instantiated, as illustrated by the example of zebra clam. In this case, the stripes bear some resemblance to those of a zebra, but their exact realization is also affected by the typical size and spatial layout of a clam. Consistent with this view, Murphy (1988) suggests that the understanding of adjective-noun combinations (e.g., long day) involves an interactive process between the adjective and noun meanings.

This instantiation view of knowledge transfer is at odds with the copy metaphor. However, the extent to which people instantiate properties is unclear. For example, when interpreting zebra clam as "a clam with stripes," do people actually construct a representation of zebra clam with stripes having aspects like those described above? During interpretation, are they sensitive to the fact that a zebra clam could not plausibly have the exact stripes of a zebra? An alternative possibility is based on the observation that knowledge of a domain is typically represented at multiple levels of abstraction (Barsalou, 1993; Marr, 1982; Stevens \& Coupe, 1978; Wisniewski \& Medin, 1994). For example, at one level of abstraction, the human body can be represented as a configuration of body parts (e.g., hand attached to arm, arm attached to torso), which in turn can have more specific representations (e.g., a hand would be represented as a configuration of fingers), which in turn can have even more specific representations (e.g., fingers represented in terms of fingernails, knuckles, and hair), and so on. In fact, frames or schemas are commonly used in cognitive psychology and AI to represent properties at multiple levels of abstraction (e.g., see Barsalou \& Hale, 1993, for a discussion). People can reason at one level of abstraction without accessing other levels. For example, people can think about washing their hands before dinner without simultaneously thinking about the fingers of their hands.

Given this view of knowledge representation, it is possible that people combine concepts by transferring fairly abstract representations of properties after doing some minimal processing for plausibility, rather than by instantiating properties. For example, the stripes of a zebra may have a fairly detailed representation, such as the one implied above, but also a more abstract representation. It is this abstract representation that is shared by zebras, barber shop poles, American flags, tigers, and so on. In understanding zebra clam, people may access the more abstract representation and assess the plausibility of a clam having stripes by noting, for example, that stripes are a type of texture, that objects have textures, and that clams are objects with textures that do not already have stripes. Thus, they may conclude that zebra clam means "a clam with stripes" and transfer the abstract sense of stripes from zebra to zebra clam. This view is consistent with the copy metaphor and does not involve the instantiation of properties. I will refer to this possibility as the abstraction view of knowledge transfer.

In the experiments that follow, I contrasted the abstraction and instantiation views of knowledge transfer in the context of novel noun-noun combinations. In particular, I examined different predictions that these views make for how people interpret novel combinations with similar constituents (e.g., zebra horse) compared with combinations with dissimilar constituents (e.g., zebra clam). All else being equal, the abstraction view does not predict a difference in how similar and dissimilar combinations are interpreted with respect to the same property. For example, according to the abstraction view, interpreting zebra horse as "a horse with stripes" and zebra clam as "a clam with stripes" would involve transferring a similar abstract representation of stripes in zebra to horse and clam.

On the other hand, the instantiation view predicts differences in how similar and dissimilar combinations are interpreted with respect to the same property. These predictions are predicated on a simple assumption: In general, the instantiation of a property in a combination (e.g., the instantiation of stripes in zebra horse) should be similar to its corresponding instantiation in the modifier concept (e.g., the instantiation of stripes in zebra) to the extent that constituents of the combination are similar (see Wisniewski, 1996, 1997).

This assumption derives from considerations of conceptual representation. In general, concepts have complex representational structure (e.g., Barsalou \& Hale, 1993; Gentner, 1983; Markman \& Gentner, 1993; Murphy \& Medin, 1985; Palmer, 1978; Wisniewski \& Medin, 1994). A particularly important aspect of these representations is the relations that capture interconnections between properties. These conceptual relations reflect the fact that properties often depend on other properties for their instantiation or realization. For example, the typical property of hammer, "for pounding in nails," depends on the hammer having a handle, being lightweight, being grasped and lifted in a certain way, having a blunt solid end, and so on. Even very simple properties, such as color, may have such dependencies. For example, it may be difficult to think of the red of a firetruck without thinking of the firetruck's shape, spatial extent, and texture.

Thus, in contrast to the abstraction view, the instantiation view predicts a difference in how similar and dissimilar combinations are interpreted with respect to the same property. Given an arbitrary property represented in a concept, the dependencies of the property are more likely to be shared by another concept to the extent that the concepts are similar. Therefore, in general, the exact sense or realization of a property in one concept will 
tend to match its realization in a similar concept. For example, the stripes of zebra horse should be more like the stripes of zebra than those of zebra clam, because the properties on which zebra stripes depend (e.g., size, shape, and texture) tend to be shared by horse and not by clam.

In the following experiments, I examined whether people instantiate properties during conceptual combination. If people instantiate properties, then they should be sensitive to different instantiations of the same property. In Experiment 1, I examined people's speeded judgments about the plausibility of interpretations of similar and dissimilar combinations that involved the same, nominally identical properties. In Experiment 2, I assessed preferences for these interpretations using a forced choice task. In Experiment 3, I directly examined the similarity between the instantiation of a property of the modifier and the instantiation of this property in the combination. In Experiment 4, I elicited interpretations of similar and dissimilar combinations. As detailed later, one would expect certain patterns of findings in these experiments if people instantiate properties. To foreshadow the results, I found evidence for instantiation during conceptual combination.

In the General Discussion, I explore possible processing accounts of instantiation in the context of conceptual combination. I also suggest that the results have important implications for models of metaphor, analogy, induction, and categorization - all of which can be broadly construed as processes involving knowledge transfer. One important implication is that instantiation may be central to these phenomena. In this regard, current models will require processes that construct new representations rather than processes that only transfer existing representations from one domain to another.

\section{EXPERIMENT 1}

Subjects made speeded judgments about the plausibility of interpretations of novel combinations. Each interpretation described a property of the modifier concept as applying to the head noun of the combination (e.g., rooster sparrow had the interpretation "a sparrow that wakes you up"). The constituents of the combination were either similar or dissimilar, such that if a property was instantiated in the combination, it would tend to match or mismatch, respectively, the instantiation of the property in the modifier. For example, compared with the instantiation of "wakes you up" in rooster television, the instantiation of "wakes you up" in rooster sparrow should better match the instantiation of "wakes you up" in rooster.

If subjects judge the plausibility of an interpretation by instantiating a property in the combination, then, under time pressure, they may be sensitive to the relative match between the instantiation of the property in the modifier and its instantiation in the combination. In particular, mismatching instantiations may lead subjects to more often judge interpretations of dissimilar combina- tions as implausible. For example, people should tend to judge "a television that wakes you up" as implausible more often for rooster television than for alarm clock television (all else being equal). In contrast, if subjects are judging whether an abstract property applies to a concept, then there should not be a difference between plausibility judgments of interpretations of similar versus dissimilar combinations.

\section{Method}

Subjects. The subjects were 75 Northwestern University undergraduates who participated as part of a course requirement.

Materials. The stimuli were 44 pairs of novel combinations and possible interpretations selected by the author. Each pair consisted of a combination whose modifier was similar to the head noun (e.g., alarm clock television) and one whose modifier was dissimilar to the head noun (e.g., rooster television), according to the author's intuitions. However, the same (i.e., nominally identical) property of each modifier could be plausibly attributed to the head noun. That is, both alarm clock television and rooster television could be interpreted as "a television that wakes you up."

Conceptually, the combinations could be divided into quadruplets. All combinations in the quadruplet could be given an interpretation involving a nominally identical property. The Appendix shows the combinations organized into quadruplets and the properties that were used to construct the interpretations of all the combinations in the quadruplet. Note that, within each quadruplet, the same modifier was paired with a similar and dissimilar head noun, and the same head noun was paired with a similar and dissimilar modifier. Thus, similar and dissimilar combinations involved identical nouns in the modifier and head noun positions. Constructing quadruplets in this manner equates for a variety of factors other than property instantiation that could account for differences between the conditions (e.g., word frequency, word length, salience of the modifier's property, the applicability of the property to the head noun).

Manipulation check. A postexperiment task assessed whether a pair of modifiers shared the property used in the interpretations involving those modifiers (e.g., whether "wakes you up" was a property that applied to both rooster and alarm clock). It was important to verify that the modifiers shared the interpretation properties. Otherwise, the results would be difficult to interpret, and the control achieved by the counterbalancing would be undermined. Twenty subjects were given the pairs of similar and dissimilar modifiers (they did not participate in the plausibility task). They examined each pair and wrote down one important property that they had in common (if there was such a commonality). The pairs were presented in one of four random orders on the left side of two sheets of paper, with a space to the right of each pair for the subject to write down a property. Each subject was randomly assigned to one of four orders.

To assess whether the modifiers shared the interpretation property, the author simply counted the number of subjects who mentioned the property as an important commonality. In a few cases, the subjects mentioned synonyms of the property. For example, given the pair boomerang and banana, the subjects mentioned not only "curved" (i.e., the interpretation property) but also "crescent shaped," "bow shaped," and "same bent shape." Synonyms were counted as tokens of the property. It was clear that the subjects considered the interpretation properties to be important commonalities shared by the modifiers. Overall, the subjects listed the interpretation property $77.3 \%$ of the time as an important property that applied to both modifiers. The interpretation property was the most frequently listed common property for 21 of the 22 pairs of modifiers ("holds things" was listed more than "has a handle" for the pair coffee cup and briefcase). Furthermore, the interpretation prop- 
erty was listed as the important commonality at least $70 \%$ of the time for 16 of the 22 pairs.

False items. Two types of false items were used. In one set (14 combinations), the interpretations referred to properties that were true of the modifier but that were asserted of a noun different from the head noun (the false head-noun items). For example, one combination was dime button, which had the interpretation "a silver penny." Here, "silver" is true of dime, but the interpretation refers to a penny and not to a button. The other set ( 14 combinations) involved properties that were not true of the modifier but that were asserted of the head noun (the false modifier-property items). For example, the interpretation of window boat was "a boat that is heavy," which asserts a property that is not true of window (i.e., "heavy") but that is true of the head noun boat. There were equal numbers of similar and dissimilar combinations among the two types of false items.

Design. The 44 pairs of test items were randomly divided into four groups of 22 combinations, subject to several constraints. First, half of the items in a group were similar combinations, and half were dissimilar combinations. Second, each group contained one combination from each quadruplet. Thus, the subject saw a specific test item property (e.g., "striped") just once. Approximately equal numbers of subjects saw each group of test stimuli. Every subject saw all of the false items ( 14 false modifier-property items and 14 false head-noun items)

Procedure. The subjects read instructions that indicated that they would see a series of novel phrases, each accompanied by a possible meaning, and that they were to read each phrase and judge whether the meaning was plausible. They were given earthquake school and the meaning "a school destroyed by an earthquake" as an example of a novel phrase and a plausible interpretation. In contrast, they were given moose mouse and "a purple mouse" and salt plate and "a box full of salt" as examples of phrases and implausible meanings. These implausible examples corresponded to the two types of false items. In the case of moose mouse, "a purple mouse" was implausible because mooses are not purple, and, therefore, it was unclear why a moose mouse would have this meaning. In the case of salt plate, the meaning was implausible because salt plate refers to a plate but its interpretation refers to a box. The subjects were told to press the "/" key (located near the lower right part of the keyboard) to indicate a plausible interpretation and the " $Z$ " key (located near the lower left part of the keyboard) to indicate an implausible interpretation.

The subjects were also instructed to respond as fast as they could while still responding accurately. In addition, the instructions noted that some meanings might be unusual but they could still be plausible. For example, moose mouse could have the unusual but plausible meaning "a mouse with antlers," although the antlers of the moose mouse would probably be much smaller than those of a moose. Finally, the instructions noted that some of the meanings might not be the ones that the subjects would give (e.g., some people think that an earthquake school is a school that teaches about earthquake safety). However, the subjects were instructed to judge whether the meaning provided was plausible.

After reading the instructions, the subject pressed a key on the computer keyboard to begin. On each trial, a cross appeared near the left side of the screen for $200 \mathrm{msec}$. It disappeared, and a combination immediately appeared, starting at the location of where the cross had been shown. After $200 \mathrm{msec}$, an interpretation appeared to the right of the combination. The subject made a judgment that cleared the screen, and the next trial began after a 2-sec delay. The combinations were presented in a different random order for each subject. The subjects were also given five practice trials before the test trials (three plausible trials and one trial of each false item type). The task took about $20 \mathrm{~min}$ to complete.

\section{Results}

The data from 2 subjects were discarded because they made errors on more than half of the trials (one subject with an error rate greater than $90 \%$ probably reversed the mapping from judgments to computer keys). Reaction time (RT) outliers were discarded on the basis of a visual inspection of the distribution of correct RTs for the test items. Times less than $500 \mathrm{msec}$ or greater than $5,500 \mathrm{msec}$ were eliminated from further analysis $(2.2 \%$ of the trials). The average RTs for interpretations of similar combinations and dissimilar combinations were 1,916 and $1,927 \mathrm{msec}$, respectively (this small difference was not statistically reliable; $t<1$ ).

As predicted, the subjects judged more of the interpretations of dissimilar combinations as implausible $(12.0 \%)$ than the interpretations of similar combinations $(5.1 \%)$. That is, under time pressure, they were more likely to say that an interpretation of a dissimilar combination was implausible (e.g., "striped tablecloth" for zebra tablecloth) than to say that an interpretation for a similar combination was implausible (e.g., "striped horse" for zebra horse). This difference was highly reliable [subjects, $t(72)=4.48$, $p<.001 ;$ items, $t(43)=3.56, p<.001] .{ }^{1}$

The subjects averaged $17.1 \%$ errors on false head-noun trials (i.e., trials on which the modifier's property was asserted of a noun that mismatched the head noun of the combination). They averaged $4.0 \%$ errors on the false modifier-property trials (i.e., trials on which the property was not true of the modifier of the combination). It is not clear how to interpret the error rate for the false head-noun trials. On first glance, this result would suggest that the subjects were judging the plausibility of interpretations by simply checking whether the property was true of the modifier. However, this interpretation would not predict the difference in the percentages of implausibility judgments between the similar and dissimilar combinations. In any event, statistical analyses revealed the same reliable difference between the similar and dissimilar test items, when the data from the subjects who committed more than $33 \%$ errors on the false headnoun trials ( 12 of 72 subjects) were discarded.

\section{Discussion}

The results suggest that subjects instantiate properties and are sensitive to differences between instantiations of the same property. The subjects were less likely to judge interpretations of similar combinations as implausible than interpretations of dissimilar combinations. These differences in judgments of implausibility mirror differences in how well the instantiation of a modifier property should match its instantiation in a combination.

Interestingly, there was no statistically reliable RT difference between the conditions. It seems plausible that, for a pair of similar concepts, the instantiation of a property in one concept would facilitate its instantiation in the second concept. For example, the instantiation of spots in 
Dalmation might indicate more clearly how to instantiate this property in poodles than it would in marbles. On this account, subjects should be faster to judge interpretations of similar combinations as plausible, since they will have completed the instantiation process sooner.

There are several reasons that may explain the lack of an RT effect. First, there could be factors that facilitated instantiation in the dissimilar combinations. To take a speculative example, although the instantiation of spots in Dalmation may indicate less clearly how spots are to be instantiated in marbles than in poodles, the actual construction of spots in marbles may take less time than in poodles perhaps because of their reduced spatial extent (see Finke, 1989, for a review of results from imagery studies that are consistent with this suggestion). Second, if property instantiation was more difficult for dissimilar combinations, the subjects might not have carried the process out to completion for these combinations. Thus, the time to instantiate properties for similar combinations could have been roughly the same as the time to partially instantiate properties for dissimilar combinations. Note that either of these accounts predicts the difference in plausibility judgments.

Several alternative interpretations for the findings are ruled out by the counterbalancing of the stimuli. First, some properties may be more applicable to one head noun than to another (e.g., spots might be applicable more to a poodle than to a marble perhaps because more animals than artifacts have spots). However, identical head nouns involving nominally identical properties were used in both similar and dissimilar combinations. Second, properties may be more salient and accessible for one modifier concept than for another (e.g., the curved shape of a boomerang may be more salient than the curved shape of a banana). However, identical modifiers involving nominally identical properties were used in both similar and dissimilar combinations. If either or both of these factors had primarily influenced plausibility judgments, then there would not have been a difference between the judgments of similar and dissimilar combinations.

On the other hand, several alternative explanations are not ruled out by the design of this experiment. First, a dissimilar combination may have had a more plausible interpretation than the one that the subjects were asked to judge. In the context of this plausible interpretation, the subjects might have decided that the experimenterprovided interpretation was implausible. Second, perhaps dissimilar combinations had more potential meanings than similar combinations, thereby increasing the subjects' uncertainty that the experimenter-provided interpretation was a plausible one. In anticipation of these possibilities, the instructions explicitly informed the subjects that there might be alternative interpretations but that they should judge the interpretation provided. Nevertheless, activation of alternative interpretations could have been automatic, and the subjects might have been unable to control their influence on plausibility judgments (Coolen, van Jaarsveld,
\& Schreuder, 1991). I address these explanations in Experiment 4 , in which the subjects generated their own interpretations of the combinations.

Although the difference in judgments of plausibility is consistent with the instantiation view, the subjects did judge a high percentage $(88 \%)$ of interpretations of dissimilar combinations as plausible. One could interpret this high percentage as indicating that subjects generally transfer abstract properties and only very occasionally instantiate properties. Thus, it is only on the rare occasion when properties are instantiated that judgments of implausibility are obtained. This view would also explain why plausibility judgments were not facilitated when the modifier and head nouns of a combination were similar. On the other hand, this high percentage may reflect the fact that two instantiations of a property often have a fair degree of similarity. For example, in judging whether "marbles with spots" was a plausible interpretation of Dalmation marbles, subjects might recognize that the spots of both a Dalmation and Dalmation marbles are black and round, even though those of Dalmation marbles are smaller and closer together. Consequently, they judge the interpretation as plausibly applying to Dalmation marbles. In the next two experiments, I attempted to provide stronger evidence that subjects instantiate properties rather than transfer abstract properties.

\section{EXPERIMENT 2}

I examined preferences for interpretations that involved nominally identical properties. In particular, a nominally identical property was associated with an interpretation of a similar combination and with an interpretation of a dissimilar combination. Both combinations had the same modifier but a different head noun that was either similar or dissimilar to the modifier. Subjects selected which of the two interpretations sounded more natural to them. As in Experiment 1, if subjects instantiate properties when they read the interpretations, then they should notice that the instantiation of the property in a similar combination more closely matches the property's instantiation in the modifier.

Given the communicative goals that motivate the production of novel combinations, subjects should prefer the interpretation of the similar combination over the one of the dissimilar combination (if they are instantiating properties). Listeners assume that speakers generally try to be accurate and clear in communicating to them (Grice, 1975), and this principle also applies to combinations (Downing, 1977). For example, suppose that you owned a black telephone. Clearly, the combination black phone more accurately applies to the phone than does gray phone, and listeners would find it odd if you used the latter in referring to your phone. In regard to Experiment 2, similar combinations more accurately apply to their referents than do dissimilar combinations (analogous to the difference between black phone and gray phone). For ex- 
ample, on reading that Dalmation poodles are "poodles with spots," subjects should create a referent poodles with spots that are very similar to those of a Dalmation (if they are instantiating properties). In contrast, on reading that Dalmation marbles are "marbles with spots," subjects should create a referent marbles with spots that are less similar to those of a Dalmation. In contrast to the spots of Dalmation marbles, the spots of Dalmation poodles will be more similar to those of a Dalmation (perhaps in terms of size, spacing, texture, and/or number). Thus, the modifier Dalmation more appropriately characterizes the poodles referred to by Dalmation poodles than it characterizes the marbles referred to by Dalmation marbles, and the interpretation of Dalmation poodles should be preferred over the interpretation of Dalmation marbles. ${ }^{2}$

Note that, relative to Experiment 1, Experiment 2 used a more sensitive measure to distinguish whether subjects instantiate properties or transfer abstract properties. In Experiment 1, the subjects judged the vast majority of interpretations of dissimilar combinations as plausible. The subjects could have used some absolute threshold of similarity and judged such interpretations as plausible because there was enough resemblance between the instantiation of the modifier property and its instantiation in the dissimilar combination. However, in Experiment 2, the subjects were encouraged to make relative judgments as they chose between two interpretations, one of which matched the instantiation of the modifier property better than the other.

\section{Method}

Subjects. The subjects were 48 Northwestern University undergraduates who participated as part of a course requirement

Materials. The stimuli were the pairs of similar and dissimilar noun-noun combinations and their interpretations used in Experiment 1 . The Appendix shows the combinations and the properties used to construct the interpretations. The pairs were randomly divided into six lists of 5 pairs and two lists of 6 pairs ( 2 pairs were omitted due to experimenter error and 4 of the combinations used were different from those of Experiment 1). ${ }^{3}$ The pairs in a list were typed on a piece of paper in a random order, with one combination and its interpretation on the left side and its corresponding combination and meaning on the right side. The combination that appeared on the left side was randomly determined, subject to the constraint that, across lists, equal numbers of similar and dissimilar pairs appeared on each side. A list was also presented in its reverse order. Therefore, this procedure yielded 16 lists of pairs and their meanings.

Only a small number of pairs was used per list so as to avoid certain response biases. With a longer list, the subjects would be more likely to notice that each pair contained a similar and dissimilar combination. The explicit awareness of this fact could have affected responding in a variety of ways (e.g., the subjects might have tried to circle only one type of combination or try to circle each type equally often).

Procedure. The subjects read brief instructions indicating that they would see some pairs of novel noun phrases and their possible interpretations. For each pair, they were to read the phrases and their corresponding interpretations and circle the one that sounded more natural to them and that they were more likely to hear in a conversation. Three randomly determined subjects completed each list. The task took several minutes to complete.

\section{Results and Discussion}

Overall, the subjects selected $70.6 \%$ of the interpretations of similar combinations as sounding more natural. This difference was highly reliable [subjects, $t(47)=11.18$, $p<.001$; items, $t(41)=9.10, p<.001]$. Thirty-five of the 48 subjects preferred interpretations of similar combinations over those of dissimilar combinations $(9$ subjects preferred the interpretations of dissimilar combinations and 4 subjects were indifferent). Of the 42 pairs of combinations, the interpretations from 30 similar combinations were preferred over those of their dissimilar counterparts (the interpretations of 6 dissimilar pairs were preferred, and there was no preference for 6 pairs). The dissimilar combinations whose interpretations were preferred over those of the corresponding similar combinations were frog carpet ("a green carpet"), sugar honey ("sweet honey"), zebra tablecloth ("striped tablecoth"), skunk mud ("smelly mud"), fish lizard ("a lizard with scales"), and boomerang cucumber ("a curved cucumber"). The corresponding similar combinations were frog toad, sugar powder, zebra horse, skunk squirrel, fish frog, and boomerang chopstick, respectively.

It is plausible that other factors besides instantiation affect a person's preference for an interpretation. Note that, for each judgment, the two choices involved the same modifiers and properties, but different head nouns. For a variety of reasons, a property might be more applicable to one head noun concept than to another. For example, the subjects strongly preferred "curved cucumber" over "curved chopstick" regardless of whether cucumber was paired with a similar modifier (i.e., banana) or a dissimilar modifier (i.e., boomerang). Perhaps curvedness more plausibly applies to cucumbers than to chopsticks because a curved chopstick would interfere with the function of a chopstick. Alternatively, perhaps people prefer "curved cucumber" because the shape dimension varies more among cucumbers than among chopsticks or because they have seen actual examples of curved cucumbers but not curved chopsticks. Factors like these, which are associated with differences between head noun concepts, cannot explain the overall preference for interpretations of similar combinations over dissimilar combinations, because each head noun appeared with both a similar and a dissimilar combination and involved the same property. Thus, if one or more of these factors primarily determined preferences, then there would be no preference for interpretations of similar combinations over those of dissimilar combinations. On the other hand, these factors could affect the magnitude of the overall preference for interpretations of similar combinations.

\section{EXPERIMENT 3}

Experiment 3 more directly measured whether subjects are instantiating properties. Subjects read the interpretation of a combination and rated the similarity of the property mentioned in the interpretation to the corresponding property of the modifier. For example, subjects 
read that Dalmation poodles were "poodles with spots" and then rated how similar the "spots of Dalmation poodles" were to the "spots of a Dalmation." The predictions are straightforward: If subjects instantiate properties, then in general they should rate the property of a similar combination and the corresponding property of its modifier as being more similar than the property of a dissimilar combination and the corresponding property of its modifier. For example, relative to the spots of Dalmation marbles, subjects should rate the spots of Dalmation poodles to be more similar to those of Dalmations. In contrast, the abstraction view does not predict a difference between the similarity of a modifier property to its corresponding property in a similar combination and the similarity of that same modifier property to its corresponding property in a dissimilar combination. In both cases, subjects transfer the same abstract representation of a property in the modifier to both the similar and the dissimilar combination.

Assuming that the instantiation hypothesis is supported, the similarity ratings provide a measure of the relative degree to which the instantiation of modifier property matches its instantiation in the combination. I then examined whether this measure is systematically related to the plausibility judgments of Experiment 1 and the preference judgments of Experiment 2, as further predicted by the instantiation hypothesis.

\section{Method}

Subjects. The subjects were 17 Northwestern University undergraduates who participated as part of a course requirement.

Materials. The stimuli were the 44 pairs of novel combinations and possible interpretations used in Experiments 1 and 2 (see the Appendix)

Procedure. The subjects read instructions indicating that a computer would present a series of phrases that they had probably not heard or seen before. Next to each phrase was one of its possible interpretations or meanings. The following example was provided:

$$
\text { chair pan: a pan with four legs }
$$

The instructions noted that this interpretation described a kind of pan (called a chair pan) that has four legs. Also, the phrase described something with a property ("has four legs") that the first thing in the phrase (chair) also had. The subjects were to read each phrase and its interpretation and then rate how similar the property of the phrase was to the property of the first thing mentioned in the phrase. In the example above, the subjects were to rate how similar they thought the four legs of a chair pan were to the four legs of a chair. The computer would present the two properties below the phrase and its interpretation after a short delay. In the example above, the subjects would see:

$$
\text { the four legs of a chair pan the four legs of a chair }
$$

The subjects were told that they would make their ratings using a number scale from 1 to 7 , with 7 indicating that the two properties were extremely similar and 1 indicating that they were not at all similar. They were told to use the numbers between 1 and 7 to indicate intermediate ratings of similarity.

The task was administered on a Macintosh LC III computer. The 88 phrases and their interpretations were presented in a random order to each subject. Each phrase remained on the screen for $200 \mathrm{msec}$. Then, the two properties appeared below the phrase and its interpretation (which remained on the screen while the subject made a rating). Below the pair of properties was a number line from 1 to 7 and the words not at all similar under 1 and extremely similar under 7 . The subjects made a rating by clicking on a number with the mouse (which boldfaced the number and increased its font size). They either clicked on OKAY to proceed to the next trial or on REDO, which restored the selected number to plain text and its previous font size, allowing the subject to make another rating. The subjects proceeded through the task at their own pace. It took approximately $30 \mathrm{~min}$ to complete.

\section{Results}

The mean similarity between the property of a modifier and the corresponding property of the combination was 4.65 for similar combinations and 3.82 for dissimilar combinations. This difference was highly reliable [subjects, $t(16)=5.76, p<.001$; items, $t(43)=7.74, p<$ $.001]$. For every subject, the average similarity rating between a modifier property and the corresponding property of the similar combination was higher than that between a modifier property and the corresponding property of a dissimilar combination. In addition, for 41 of the 44 modifier nouns, the average similarity rating between its property and the corresponding property of the similar combination was higher than that between the property and the corresponding property of the dissimilar combination. The only exceptions were giraffe, sugar, and boomerang. Their properties, "long neck," "sweet," and "curved," respectively, were rated as more similar to the corresponding properties for giraffe duck, sugar honey, and boomerang cucumber than to those for giraffe horse, sugar powder, and boomerang chopstick, respectively.

In Experiments 1 and 2, I argued that plausibilty and preference judgments should be affected by the relative match between the instantiation of a property in the modifier and its instantiation in the combination. Because the similarity ratings provide a direct measure of this match, they should reliably correlate with the preference and plausibility judgments. These predictions were supported by the data. There was a correlation of .65 between the similarity of a modifier property to the corresponding property of a combination and the percentage of time (in Experiment 1) that the property was judged to plausibly apply to the combination.

To examine the correlation between similarity ratings and preference judgments in Experiment 2, I calculated for each similar combination the difference between the similarity of its modifier property to the corresponding property of the similar combination and the similarity of that modifier property to the corresponding property of the dissimilar combination. For example, the difference score for sugar powder was -1.12 , reflecting the difference between the similarity of the sweetness of sugar to the sweetness of sugar powder (3.41) and the similarity of the sweetness of sugar to the sweetness of sugar honey (4.53). There was a correlation of .61 between the difference score of a similar combination and the percentage of time in Experiment 2 that the subjects preferred its interpretation to the corresponding one of the dissimilar combination. 
I also examined the relationship between extreme values of similarity judgments and plausibility and preference judgments. Extreme values of similarity should correspond to extreme differences between instantiations of the same property, which in turn should show correspondingly strong effects on plausibility and preference judgments. These predictions were supported by the data. The 10 combinations whose interpretations involved properties that were most dissimilar to those of their modifiers had an average similarity rating of just 2.73 (the average similarity rating between all modifier properties and properties of combinations was 4.2). The percentage of time in Experiment 1 that the subjects judged the interpretations of these combinations as implausible was $26.9 \%$ (the average percentage of implausibility judgments for all interpretations was $8.6 \%$ ). In contrast, the 10 combinations whose interpretations involved properties that were most similar to those of their modifiers had an average similarity rating of 5.55 . The percentage of time in Experiment 1 that the subjects judged the interpretations of these combinations as implausible was less than $1 \%(0.55 \%)$.

I also determined the 10 similar combinations with the smallest difference scores (as described above). The average difference score for these 10 combinations was just .08 (compared with .83 for all similar combinations), which indicates that the instantiation of a modifier property in the similar combination had about the same degree of match as the instantiation of that property in the dissimilar combination. Corresponding to this very small difference score, the subjects in Experiment 2 preferred the interpretation of the similar combination over the dissimilar combination just $46 \%$ of the time (basically, they were indifferent between the interpretations, as would be expected). Recall that overall, the subjects preferred the interpretation of the similar combination $70.6 \%$ of the time. In contrast, the 10 similar combinations with the highest difference scores had an average score of 1.69 . The subjects preferred the interpretations of these similar combinations $84.9 \%$ of the time.

\section{Discussion}

The results suggest that on reading an interpretation of a novel combination that mentioned a property of the modifier, the subjects instantiated that property in the combination rather than transferred an abstract representation of the property. An instantiation of a property in the combination was more likely to match the instantiation in the modifier when the head noun and modifier concepts had similar representations. Thus, the subjects rated a property of a combination as more similar to the modifier property when the constituents of the combination were similar.

The results also suggest that the subjects instantiated properties in Experiments 1 and 2 and that their preferences for interpretations and judgments about their plausibility were influenced by how well the instantiation of a property in the modifier matched its instantiation in the combination. There were fairly strong positive relationships between the similarity ratings of properties in Experiment 3 and the plausibility of interpretations in Experiment 1 and between similarity ratings and the relative preferences for interpretations in Experiment 2.

\section{EXPERIMENT 4}

In Experiment 4, I examined the interpretations that subjects gave to the similar and dissimilar combinations. The claim that people instantiate properties during conceptual combination makes subtle predictions about how they will phrase their interpretations. One prediction is that subjects should tend to explicitly describe a property of the combination as (also) belonging to the modifier or as resembling one of the modifier to the extent that it applies in the same way to both terms- that is, to the extent that it is instantiated in the same way. Therefore, this way of phrasing interpretations should be more likely for similar combinations. For example, consider again the interpretations of zebra horse and zebra clam. The instantiation of stripes in zebra horse should result in stripes that are quite similar to those of a zebra, whereas the instantiation of stripes in zebra clam should not. Assuming that subjects follow cooperative principles of communication, this difference should be reflected in their phrasing of these interpretations. In particular, if subjects try to phrase their interpretations accurately, then they should tend to say that a zebra horse is "a horse with the stripes of a zebra," "a horse with zebra stripes," or "a horse with stripes that look like those of a zebra." In contrast, for dissimilar combinations (e.g., zebra clam), they should be less likely to explicitly note a resemblance between a property of the combination and one of the modifier (because, in many cases, the instantiations will not strongly resemble each other).

A second prediction is that subjects should provide more elaborate descriptions of properties that have been instantiated in dissimilar combinations. For example, the instantiation of stripes in zebra clam should be a new version of stripes that differs from zebra stripes. If subjects try to phrase their interpretations accurately, then they should refer to these differences. Also, because the instantiation of a property in a dissimilar combination should share some (but not all) commonalities with the modifier property, subjects might also indicate which commonalities are shared (e.g., they might note that a zebra clam has black and white stripes).

The interpretations were also examined to assess alternative explanations for the findings in Experiments 1 and 2. Compared with the similar combinations, dissimilar combinations might have had a salient, plausible interpretation (which was not the interpretation that the subjects judged) or they may have had more potential interpretations. In the context of a salient, alternative interpretation or many potential interpretations, the subjects 
might have been less likely to judge the experimenterprovided interpretation as plausible and less likely to prefer this interpretation.

\section{Method}

Subjects. The subjects were 22 Northwestern University undergraduates who participated as part of a course requirement

Materials. The stimuli were the pairs of similar and dissimilar noun-noun combinations shown in the Appendix. The pairs were divided into two lists of 22 combinations by randomly assigning one of the pairs in a quadruplet to one list and the other pair to the second list. Thus, the pairs in a list shared the same modifier, but, for one combination, the head noun was similar to the modifier, and, for the other combination, it was dissimilar.

Procedure. The subjects read instructions indicating that they would see some novel noun-noun phrases and that, for each phrase, they should type a description of its most plausible meaning. (The phrases were presented on the computer screen in a spreadsheet.) The subjects were instructed to assume that they had heard the novel phrases in a conversation, and they were to think of what the person meant when he/she said the novel phrase. The task was illustrated by way of an example that involved earthquake school and a variety of plausible meanings: "a school that was destroyed by an earthquake," "a school that teaches people about safety steps for surviving earthquakes," and "a school that has a lot of turmoil." The subjects were also instructed to avoid vague responses (e.g., "a school that has something to do with earthquakes"). After reading the instructions, a spreadsheet was opened that contained the phrases. The subjects typed in their interpretations at their own pace. The task took about 30 min to complete. Half (11) of the subjects saw the first list of combinations, and half saw the second list. A list was presented in a random order for 6 of the subjects and in its reverse order for the other 5 subjects.

Scoring. The interpretations for each combination were gathered into a group. A research assistant then examined each interpretation and simply judged whether it involved a property of the modifier being asserted of the combination. She did not know the purpose of the experiment. Examples of these interpretations included "a swimming lizard" for fish lizard, "a porpoise that has a long snout like an elephant" for elephant porpoise, "a bird that wakes you up every morning" for alarm clock sparrow, "water with a slight coffee flavor" for coffee water, and "a duck which is quite large and exhibits features and characteristics of a goose" for goose duck.

Consistent with previous research, the subjects interpreted combinations using two other strategies (Wisniewski, 1996; Wisniewski \& Markman, 1993). One strategy was to assert that a relation held between the constituents of the combination. Some examples included "a mouse that lives on an airplane" for airplane mouse, "a pig that feeds on cacti" for cactus pig, and "a rope that is made of yarn" for yarn rope. Another (less frequent) strategy was to interpret a combination as referring to a hybrid of the constituents, as in "a hybrid descendent of zebras and horses" for zebra horse and "a mix between a frog and a toad" for frog toad. The subjects also occasionally gave responses that did not conform to the instructions, such as "no idea" for banana chopstick and "a red fire-engine" for firetruck car. One subject's data were discarded because he or she misinterpreted the task and primarily listed commonalities between constituents

For the interpretations that involved properties, the author and research assistant independently judged whether an interpretation included the property involved in the experimenter-provided interpretations or strongly implied the presence of that property. Examples of the latter interpretations were "a banana that looks like a chopstick" for banana chopstick (implying that it is curved) and "a tablecloth with the American flag design" for American flag table- cloth (implying that it has stripes). The research assistant and author agreed on $94.9 \%$ of their judgments. Differences were resolved through discussion

For all interpretations that were based on the property of the experimenter-provided interpretation, the research assistant and author judged whether they explicitly described a property of the combination as (also) belonging to the modifier. For example, the interpretations given above for coffee water, elephant porpoise, and goose duck describe a property as explicitly belonging to the modifier, in contrast to the interpretations given above for fish lizard and alarm clock sparrow. The research assistant and author agreed on $99 \%$ of their judgments. Differences were resolved through discussion. Then, the author examined the remaining interpretations and judged whether they provided more elaborate descriptions of properties. Examples of these interpretations included "poodles with small dots" and "poodles which have spots on their skin" for dice poodles, which are instantiations derived from "has spots" in dice, and "a flower that has sharp petals" and "a yellow flower with spikes growing out of it" for porcupine dandelion, which are instantiations derived from "prickly" in porcupine.

The author and research assistant also examined whether the dissimilar combinations had salient interpretations that might have competed with the experimenter-provided interpretations in Experiments 1 and 2 . They independently read through the interpretations of a combination and selected the consensus property or relation. In this case, the consensus property or relation was arbitrarily considered to be the most common one mentioned by at least 3 subjects (out of a possible 10 or 11). The consensus property or relation, rather than interpretation, was selected because interpretations sometimes included multiple properties, as in "a pear that is round and red like an apple" for apple pear and "a robin that is black and makes the same sound as a crow" for crow robin. Overall, the raters agreed on $92 \%$ of their judgments. They resolved their differences through discussion.

Finally, the research assistant divided the interpretations of a combination into distinct types and occurrences of those types (i.e., tokens). A second research assistant also performed this task (she also did not know about the purpose of the study). The average number of types for the combinations was 5.59 for the first research assistant, compared with the 6.76 for the second research assistant. Although, the second research assistant tended to judge the combinations as having more types, there was a reliable correlation between the judgments of the two raters (.73). For 77 of the 88 of the combinations $(87.5 \%)$, the raters' judgments of the number of types differed by 2 or less.

\section{Results}

Both the similar and the dissimilar combinations had comparable numbers of interpretations that mentioned the property used in the experimenter-provided interpretations of Experiments 1-3. By the author's count, $44.2 \%$ of the similar combinations mentioned or strongly implied the property, as did $41.3 \%$ of the dissimilar combinations. These percentages were $37.4 \%$ and $37.7 \%$, respectively, by the first research assistant's count. As predicted, the percentage of these properties that were explicitly described as belonging to the modifier was higher for similar combinations than for dissimilar combinations $(33.7 \%$ vs. $19.1 \%$, respectively). This difference was highly reliable [subjects, $t(20)=4.05, p<.001] .^{4}$

Also as predicted, the subjects tended to provide more details about the experimenter properties for the interpretations of dissimilar combinations than for those of 
Table 1

Interpretations of Dissimilar Combinations That Imply That the Modifier Property Is Being Instantiated Differently in the Combination (Experiment 4)

\begin{tabular}{lll}
\hline \multicolumn{1}{c}{ Combination } & Modifier Property & \multicolumn{1}{c}{ Interpretation } \\
\hline alarm clock sparrow & wakes you up & $\begin{array}{l}\text { a bird that wakes you up in the } \\
\text { morning with an annoying chirp } \\
\text { a television with an automatic }\end{array}$ \\
rooster television & wakes you up & $\begin{array}{l}\text { alarm integrated into it } \\
\text { an onion which has a reddish colored skin }\end{array}$ \\
firetruck onion & red & a bus that can take off and fly \\
bird bus & flies & a mouse with wings \\
airplane mouse & flies & deep black murky water \\
crow water & prickly & a pig that is covered with a lot of prickly hair \\
cactus pig & prickly & a flower that has sharp petals \\
porcupine dandelion & sticky & ribbon that has glue already on the back of it \\
glue ribbon & pointy & a roof composed of long pointed logs \\
pencil roof & fast & a dog that can run very quickly \\
race car dog & long neck & a duck with a deformed neck \\
giraffe duck & & .
\end{tabular}

the similar combinations $(45.2 \%$ vs. $26.3 \%$, respectively). This difference was highly reliable [subjects, $t(20)=4.18$, $p<.001]$. Table 1 presents some of these elaborations. They provide enough detail to suggest how the property of the modifier was differentially instantiated in the head noun of the combination.

There was no evidence that, relative to the similar combinations, the dissimilar combinations had a highly salient interpretation that competed with the experimenter-provided interpretation in Experiments 1-3. In examining the interpretations, it was apparent that the property used in the experimenter-provided interpretations often was the most common one mentioned in the interpretations for a combination. This result obtained even though the property was mentioned in only about $40 \%$ of the subject-generated interpretations. More specifically, for 28 of the similar combinations and 26 of the dissimilar combinations, the consensus property was the property used in the experimenter-provided interpretations of Experiments 1-3. Thus, for a majority (and about the same number) of similar and dissimilar combinations, the consensus property was the property used in the experimenter interpretations. For the remaining combinations, it also did not appear that the dissimilar combinations had alternative interpretations that were more salient than those of the similar combinations (as measured by production frequency). Twelve of the $16 \mathrm{sim}$ ilar combinations had consensus properties or relations, and they occurred an average of 4.17 times. Seventeen of the 18 dissimilar combinations had consensus properties or relations, with an average of 4.06 occurrences. ${ }^{5}$ Some examples of these consensus properties or relations included "yellow" (which was mentioned in 7 interpretations of banana cucumber), "toad that lives in the grass" (a relation mentioned in 7 interpretations of grass toad), "cold" (a property mentioned in 5 interpretations of snow soda), and "box that holds a coffee cup" (a relation mentioned in 8 interpretations of coffee cup box).

There also was no evidence that the dissimilar combinations had more different kinds of interpretations than did the similar combinations. By the first research assistant's count, there were on average 5.45 different kinds of interpretations for the dissimilar combinations and 5.72 for the similar combinations. These averages were 6.57 and 6.95 , respectively, by the second research assistant's count. The small differences were not statistically reliable or in the direction expected if dissimilar combinations had more potential interpretations [for the first research assistant, $t<1$; for the second research assistant, $t(43)=1.06, p<.30]$.

\section{Discussion}

The subjects phrased their interpretations of novel combinations in ways that suggested that they instantiated a property of the modifier in the combination. Two aspects of the phrasings provide evidence for instantiation. First, for similar combinations, the subjects were more likely to explicitly refer to a property as resembling or being the same as a property of the modifier. One would expect this result if the subjects were instantiating properties in the combination, because an instantiation of a property in a combination is more likely to resemble its instantiation in the modifier when the constituents of the combination are similar.

Second, for dissimilar combinations, the subjects were more likely to modify a property in a way that suggested that its instantiation in the combination differed from its instantiation in the modifier concept. To illustrate, consider some examples from Table 1. The interpretation "a bird that wakes you up with an annoying chirp" (given for alarm clock sparrow) suggests that the subject instantiated "waking up" in sparrow rather than transferred an abstract sense of "waking up" from alarm clock. It describes a mechanism specific to sparrow that realizes this property (i.e., chirping in an annoying way). As another example, consider "a bus that can take off and fly" for bird bus. This interpretation suggests that the subject had a specific sense of flying in mind that was compatible with bus (probably by analogy to airplane) and did not transfer an abstract sense of flying from bird. As a 
final example, consider "a pig covered with a lot of prickly hair" for cactus pig. Again, this interpretation suggests that the subject had a sense of prickliness in mind that was specific to pig - one realized through prickly hairs that cover the pig's body.

These results provide further evidence that people do not interpret combinations only by using a general mechanism that transfers abstract properties from the modifier to the combination. However, the findings do not rule out the possibility that sometimes the subjects transferred abstract properties. In $46 \%$ of the interpretations involving experimenter properties for the dissimilar combinations, the subjects simply mentioned the property and did not provide details about its instantiation. However, this percentage likely underestimates the tendency of the subjects to instantiate properties. For one thing, people typically create a noun-noun combination in order designate an important subcategory that contrasts with the head noun category (Downing, 1977; Murphy, 1988; Wisniewski, 1997). For example, someone might coin the phrase giraffe duck to designate a subcategory of duck that differs from other ducks in having an exceptionally long neck. In doing so, people formulate an interpretation relative to the head noun category and not to the modifier. Thus, their interpretation will highlight differences between the combination and head noun category rather than differences between the combination and modifier. For example, the neck of a giraffe duck may primarily differ from that of a duck in being much longer, and, thus, a person will produce the interpretation "a duck with a very long neck." This interpretation does not capture how the instantiation of long neck in giraffe duck differs from its instantiation in giraffe since it was phrased in order to contrast the neck of a giraffe duck with that of a duck. However, the instantiation of long neck in giraffe duck may considerably differ from the instantiation of long neck in giraffe (the neck of a giraffe duck will not be as long or as thick as that of a giraffe).

In addition, there may be subtle differences between the instantiation of a property in the combination and that in the modifier. However, subjects may not be able to articulate those differences, or they may assume that they are obvious and need not be mentioned. For example, according to the instantiation view, the red in firetruck onion differs from the red in firetruck at least in its spatial extent. However, this difference may be awkward for subjects to articulate, or they may assume that it is common knowledge that an object's color often applies to its entire exterior. Thus, they do not bother mentioning this common knowledge in their interpretation.

Finally, the results argue against several alternative explanations for the findings of Experiments 1 and 2. In particular, relative to the similar combinations, dissimilar combinations might have had a salient, plausible interpretation or more potential interpretations that contrasted with the experimenter-provided interpretations. In the context of a salient, alternative interpretation or many potential interpretations, the subjects might have been less likely to judge the experimenter interpretation as plausible and less likely to prefer this interpretation. However, analyses of the interpretations suggested that dissimilar combinations had neither alternative interpretations that were more frequent than those of similar combinations nor more possible interpretations.

\section{GENERAL DISCUSSION}

Taken together, the results of these experiments suggest that people combine concepts by instantiating properties. I define instantiation as the use of a representation of a property that is specific to one concept to construct a new version of that property that is specific to the second concept. In general, the new version differs from the source property to the extent that the concepts in which these properties are embedded are also different. A property depends on other properties in a concept for its exact realization, and, to the extent that two concepts are different, they will not share these dependencies. Thus, the property will generally not have the same instantiation in two different concepts. The results are inconsistent with the view that people combine concepts only by accessing an abstract representation of a property in the modifier and transferring it to the combination.

A variety of findings support the instantiation view. In Experiment 1, the subjects were more likely under time pressure to judge that an interpretation of a similar combination was plausible than they were to judge that an interpretation of a dissimilar combination was plausible, even though both interpretations involved the same property. In Experiment 2, the subjects generally preferred an interpretation of a similar combination over one of a dissimilar combination, even though both interpretations involved the same property. These findings suggest that when subjects read an interpretation of a combination that refers to a property of the modifier, they instantiate that property in the head noun of the combination. In general, the instantiation of a property in the modifier will match its instantiation in the head noun when the modifier and head noun are similar. Thus, the modifier of a combination more appropriately captures the meaning of a combination when it is similar to the head noun, leading to the advantages in plausibility and preference for interpretations of similar combinations.

The results of Experiments 3 and 4 provide more direct evidence of instantiation. In Experiment 3, the subjects tended to rate the property of a similar combination and its counterpart in the modifier as being more similar than the property of a dissimilar combination and its counterpart. This result would be expected if subjects were instantiating properties in the combinations: The similarity of an instantiated property in the combination to its instantiation in the modifier should be higher if the modifier and head noun are similar. The result would not be expected if subjects transfer only an abstract representation from the modifier concept to the head noun concept because the abstract property will be identical whether it 
is transferred to a similar combination or a dissimilar combination.

In Experiment 4, the subjects generated their own interpretations of similar and dissimilar combinations. For similar combinations, the subjects were more likely to explicitly note that a property mentioned in their interpretation resembled the corresponding property of the modifier. This finding would be expected if subjects were instantiating properties because the instantiations of properties of similar combinations are more likely to resemble the corresponding properties of their modifiers. For dissimilar combinations, the subjects were more likely to describe a property in a way that suggested that its instantiation in the combination differed from its instantiation in the modifier concept. This finding would be expected if subjects were instantiating properties of dissimilar combinations because the instantiations of properties of dissimilar combinations are less likely to resemble the corresponding properties of their modifiers.

The present findings are consistent with recent work on the verification of object properties. Solomon and Barsalou (in press) had subjects verify object-part pairs (e.g., horse-mane) after earlier verifying a nominally identical part that was either similar (e.g., pony-mane) or dissimilar (e.g., lion-mane). Subjects were faster and made fewer errors in verifying that a part belonged to an object if it had been preceded by a similar instantiation of that part. Solomon and Barsalou's interpretation of these findings coincides with the one given for the present results: People are sensitive to the instantiations of properties with the relative match between property instantiations affecting judgments. The present experiments differ in that they examined how an existing property of an object was instantiated in a second object that was not previously characterized by that property. Often, the instantiation resulted in a "novel version" of the property (e.g., the "prickly" property of cactus was instantiated in pig as "covered with a lot of prickly hair" or "having sharp skin").

In the remainder of this paper, I revisit several issues in more detail. First, I describe some plausible mechanisms that carry out instantiation, and I examine a number of issues for further research. Second, I relate the instantiation view to models of analogy, metaphor, conceptual combination, and induction. I will suggest that most of these approaches subscribe to a copy metaphor of knowledge transfer and that this view must be abandoned if we are to make significant progress in capturing how people use knowledge in one domain to understand another.

\section{Mechanisms of Property Instantiation}

People appear to instantiate properties using at least two mechanisms. One mechanism is a comparison and construction process (Wisniewski, 1997). In comparison, people align the modifier and head noun concepts. The alignment puts common parts of the representations into correspondence, which then suggest where in the head noun a new property is to be constructed and which properties in the head noun are relevant to its construction. To take a simplified example, suppose that someone learns about a new type of horse called a zebra horse, which has stripes. In attempting to construct the meaning of zebra horse, a person might put the representation of a horse's body into correspondence with the representation of a zebra's body because they are similarly shaped and because they have similar conceptual relations to similar components (e.g., similar, vertically oriented legs are connected to a similar underside of the body at similar places). In placing the body and neck of horse into correspondence with the body and neck of zebra, the comparison process has determined where the stripes can be constructed in zebra horse. In particular, they should run alongside the body and neck of a horse just as they do in a zebra. Furthermore, the comparison process indicates which properties of the head noun are relevant to constructing the stripes. For example, the exterior of a horse's body is relevant to constructing the stripes but not its teeth, tail, what it typically eats, and so on.

Comparison appears necessary in determining where a property is to be constructed in the head noun of the combination. For example, people know that if a zebra horse was a horse with stripes, then it would have stripes on its body. The fact that horses have bodies just as zebras do allows people to infer that the stripes of a zebra horse would also be on its body. The comparison process determines this correspondence between the (striped) body of a zebra and the body of a horse.

One might argue against the necessity of such a comparison process by suggesting that people determine where a property should be constructed in a concept by some general reasoning mechanism. For example, people might reason that a zebra horse has stripes on its body because striped things generally have stripes on their exteriors. However, intuition suggests that such reasoning strategies do not have sufficient generality. For example, if a zebra had stripes only on its face, then a zebra horse would probably have stripes only on its face. In this case, a comparison process enables the derivation of this interpretation by determining a correspondence between the face of a horse and the face of a zebra. In addition to this necessity argument, there is empirical evidence that people employ this process during conceptual combination (Wisniewski, 1996; Wisniewski \& Love, 1998).

Property instantiation is complicated because the comparison process must sometimes align quite different mental representations whose commonalities may not be as obvious as those in the example of zebra horse. Furthermore, instantiation may involve the construction of a property that is quite different from the source (modifier) property. For example, consider how people might interpret zebra clam. They presumably align body of zebra with shell of clam, even though these representational elements are much less similar than are the bodies of zebra and horse. This correspondence might occur because both the body of a zebra and the shell of a clam indicate the major axes of a zebra and a clam, respectively. 
The resulting alignment suggests that the stripes of a zebra clam should be on the exterior of its shell (just as the stripes of a zebra are on the exterior of its body). However, a zebra clam (in contrast to a zebra horse) cannot have stripes that are highly similar to those of a zebra. The issue then is how the stripes are constructed.

Wisniewski (1997) suggests two general constraints on construction, which follow from basic communicative goals of using noun-noun combinations. First, the new version of a property must bear some resemblance to its source property in the modifier. A speaker who uses zebra clam to refer to a clam whose stripes have little resemblance to those of a zebra would confuse listeners. Second, the combination that results from instantiating the modifier property in the head noun must still be a type of the head noun category. For example, the stripes of a zebra clam must not alter people's concept of clam so much that they would no longer consider a zebra clam to be a clam. Imagine constructing the stripes of a zebra clam so that they bore a striking resemblance to those of a zebra. In this case, the representation of clam would have the shape and size of a zebra but would not refer to anything that we would call a clam. Thus, property instantiation can be viewed as a compromise between two competing constraints. The instantiated property must resemble the source property of the modifier, but the resulting combination must be a type of the head noun category. Wisniewski (1997) details examples of people's interpretations that follow these constraints.

This account of instantiation assumes that people construct a new representation that corresponds to the interpretation of the combination. However, people may also access an existing representation referring to a realworld entity that matches the interpretation of the combination. For example, in Experiment 4, two of the interpretations of grass carpet were "astroturf." Thus, instantiation may arise through a second mechanism called extensional feedback that reflects our knowledge of actual things in the world (Hampton, 1987; Murphy, 1988). Of course, the use of extensional feedback to instantiate a property is compatible with the present findings. For example, suppose that people retrieve a particular instance of a red car in interpreting firetruck car and a particular instance of a red onion in interpreting firetruck onion. Relative to the redness of the onion, the redness of the car should be more similar to the redness of a firetruck (perhaps in terms of spatial extent, reflectance, etc.).

The use of extensional feedback is supported by many studies showing that an adjective takes on different meanings or instantiations depending on the noun that it modifies (Halff, Ortony, \& Anderson, 1976; Heit \& Barsalou, 1996; Medin \& Shoben, 1988; Murphy, 1988; Rips \& Turnbull, 1980). Explanations of this effect tend to focus on extensional feedback. For example, Medin and Shoben (1988, Experiment 2) found that the similarity between the same pair of adjectives changed with the noun. In one case, subjects considered gray clouds and black clouds as more similar than gray clouds and white clouds. However, this relationship reversed when the noun was hair: gray hair and white hair were more similar than gray hair and black hair. This finding suggests that people access knowledge about actual clouds and hair. Gray and black clouds are more similar because they are both associated with a change of state to stormy weather, in contrast to white clouds. However, gray hair and white hair are more similar because they are both associated with aging, in contrast to black hair. As a second example, Halff et al. (1976) had subjects rate the similarity of red in different sentence contexts (e.g., "The fire is red hot" vs. "As the sun set the sky turned red"). They concluded that the representation of red varied along a real-valued interval of redness and depended on the particular instances of $r e d$ that people retrieved that were cued by the sentence context.

In the present experiments, the subjects might have employed both the comparison/construction mechanism and the extensional mechanism since the novel combinations varied in terms of whether they did or did not have real-world referents. To show this, 12 subjects were given the interpretations used in Experiments 1-3 in one of two random orders and were instructed to circle the things that they had "seen or heard of more than a couple times." (They did not participate in any of the experiments.) For half of the entities described by the interpretations, less than half of the subjects reported encountering the entity more than a couple times. (Overall, an average of $48.7 \%$ of the subjects reported not encountering a given entity.) For example, 2 or fewer subjects claimed to have encountered black water, a black robin, a curved chopstick, a spider that stings, a shark with scales, poodles with spots, a prickly dandelion, and a prickly pig. There also were entities that most subjects reported having encountered (11 or more subjects claimed to have seen or heard of a green carpet, a bowl with a handle, a box with a handle, a pointed roof, white sand, a round pebble, thin rope, and a striped tablecloth).

Thus, instantiation appears to be carried out by at least two mechanisms. One mechanism compares the modifier and head nouns and aligns their commonalities in order to determine where in the head noun the property is to be instantiated. A new version of the property is created on the basis of constraints provided by both the modifier and the head noun concepts. A second extensional mechanism is important when novel combinations have real-world referents. It involves the retrieval of existing representations that correspond to actual entities named by the combination. Note though that the two mechanisms may be interdependent. For example, the comparison/construction process may initially produce a representation that is used as a retrieval cue by the extensional mechanism.

The present account of instantiation raises a number of issues for further research. First, this account describes instantiation mechanisms only at a general level, and it does not identify more specific mechanisms involved in instantiation. For example, the present account 
does not specify the exact details of the construction process (i.e., mental operations that create a property, the input to these operations, and the representation of the property that results from these operations). The lack of a more specific processing account is due to the nature of the present experiments. They were designed to establish that people do instantiate properties associated with nouns and to examine the effects of concept similarity on instantiation. They did not examine in detail how people instantiate properties.

Second, the instantiation account does not preclude the possibility that people transfer abstract representations. Perhaps the tasks used in the present experiments created situations that especially encouraged instantiation. For example, in Experiment 2, the subjects had to choose whether they preferred an interpretation of a similar combination or one of a dissimilar combination, which involved the same property (and, hence, would be expected to have the same abstract representation of that property). Thus, this task might have encouraged the subjects to instantiate the properties in order to give them some basis for making a choice. One might pursue this view further and suggest a processing account in which people initially transfer abstract representations of properties, employing instantiation when a task or context encourages such a process. For example, a person may initially interpret $z$ ebra clam as a clam with an abstract property of "having stripes" but later employ instantiation when encountering an actual zebra clam that visually indicates the instantiation.

Finally, my account of instantiation has addressed only combinations in which a property of the modifier noun is instantiated in the combination. Many combinations are not interpreted in this way, including adjectivenoun combinations and noun-noun combinations that involve a relation between the modifier and the head noun of the combination. An example of the latter type is shoe box, which refers to a box that contains shoes and not to a box with a property of a shoe. Recently, Wisniewski and Love (1998) found that the production frequency in English for noun-noun combinations with relation interpretations was about $70 \%$, relative to $30 \%$ for combinations with property interpretations.

Clearly, instantiation is involved in the interpretations of these other kinds of combinations. As previously noted, the instantiation of an adjective's meaning depends on the noun that it modifies (e.g., boiled celery is soft, but a boiled egg is hard). Combinations with relation interpretations also involve instantiation. For example, to interpret ladder box as "a box that contains ladders," people may modify box so as to conform to the shape of a typical ladder and to open from the side as opposed to the top. Thus, the exact sense of box will differ from its sense in other combinations (e.g., shoe box; see Cruse, 1986 , p. 52, and Murphy, 1988, for related discussions). These other kinds of combinations may be understood through the extensional mechanism when they name real-world referents. However, it is presently unclear how they are understood when they do not have real-world referents. The comparison/construction mechanism does not apply to these cases.

\section{Models of Knowledge Transfer}

This characterization of property instantiation has important implications for models of metaphor, analogy, conceptual combination, and induction. In a broad sense, these models attempt to characterize how people use knowledge in one domain to understand another domain.

Metaphor and analogy. I have argued that instantiation involves both comparison and construction processes. Current models of metaphor and analogy have emphasized the importance of one or the other of these processes, but not both (Wisniewski, 1997). The notion that comparison is important in conceptual combination was actually inspired by several models in which comparison is considered central to how metaphors and analogies are understood. These models include the structure mapping engine (Falkenhainer et al., 1989; Gentner, 1983, 1989) and the analogical constraint mapping engine (Holyoak \& Thagard, 1989). ${ }^{6}$ As noted, comparisons involve finding correspondences between concepts. However, determining these correspondences is potentially a computationally intractable problem. As a result, these models have focused on the psychological factors that constrain the comparison process (see Holyoak \& Thagard 1989, for discussion). However, these approaches view knowledge transfer as a straightforward process in which a predicate of one domain is copied to a second domain and its arguments replaced by those of the second domain (see, e.g., Gentner, 1983, p. 159; Holyoak \& Thagard, 1989, p. 297; Hummel \& Holyoak, 1997).

Another view of metaphor understanding is the interactive property attribution approach (Glucksberg \& Keysar, 1990; Glucksberg, Manfredi, \& McGlone, 1997), which acknowledges the importance of the construction aspect of instantiation. For example, in earlier work on which this approach is based, Glucksberg, Gildea, and Bookin (1982) noted that the same properties of one concept are differentially instantiated in different domains:

The statement $X$ is a butcher can always be taken to mean
that $\mathrm{X}$ is negatively evaluated, and that $\mathrm{X}$ is grossly and
characteristically incompetent as well. The particular way
that $\mathrm{X}$ 's incompetence is instantiated will depend on who
or what $\mathrm{X}$ might be. If $\mathrm{X}$ is a surgeon, the incompetence
takes the form of botched operations, with bleeding, dis-
figurement, and death the likely consequences. If a pianist
is a butcher, then the incompetence is not merely the for-
getting of certain parts of the piano pieces or the lack in
the repertoire, but that the music is plowed through insen-
sitively, too loudly, without any hint of subtlety or beauty.
(p. 95 )

In these examples, the authors imply that one concept provides information that acts as a source for the construction of new properties in a second. Thus, metaphors 
are not understood by copying over predicates and replacing arguments, as implied by other approaches.

However, the property attribution approach does not specify a role for comparison in metaphor understanding. As I have argued, though, comparison is necessary in order to find correspondences between concepts, which in turn indicate where new properties are to be constructed and which existing properties are relevant to their construction. For example, in understanding "that surgeon is a butcher," people may note the correspondences between "hands," "scalpel," and "human body" in surgeon and "hands," "knife," and "meat" in butcher, respectively. The former properties are involved in constructing the sense in which a surgeon is incompetent.

Specifying how properties are instantiated in understanding metaphors is especially challenging because metaphors prototypically involve highly dissimilar concepts (e.g., that relationship is a seesaw, the ballerina was a butterfly, that dinner was a roller coaster). Thus, metaphors generally cannot be understood by straightforward transfer of predicates from one domain to another (cf. zebra horse) because the dependencies associated with a predicate are not present in the other domain and the predicate cannot be applied. For example, undergraduates often interpret "that dinner was a roller coaster" as "a dinner with courses that alternate between tasting good and bad" (Wisniewski, 1997). Clearly, the source property for this interpretation (perhaps "roller coasters going up and down") cannot be directly applied to dinner, and its subsequent instantiation in dinner is very different from the source (though people easily interpret this metaphor). Furthermore, as noted, finding correspondences between domains is necessary in order to instantiate a property; however, determining these correspondences in metaphors is less straightforward because representational elements are usually so dissimilar. For example, Wisniewski (1997) argued that part of understanding "that dinner is a roller coaster" involves putting very different predicates into correspondence: for example, aligning a height dimension in which a person is relatively high to the ground or low to the ground (in roller coaster) with a taste dimension in which food either tastes good or bad (in dinner). Wisniewski (1997) describes a number of factors that influence how people put such nonidentical predicates into correspondence (see also Lakoff \& Johnson, 1980; Marks \& Bornstein, 1987; Ortony, 1979).

Conceptual combination. In general, the present findings lie outside the bounds of current models. Several approaches focus on how noun-noun combinations are interpreted with relations rather than properties, as in the present experiments (Gagne \& Shoben, 1997; Murphy, 1988). For example, in the concept specialization model (Murphy, 1988), a concept is viewed as a schema or frame. The frame represents knowledge about an entity in the form of slots and fillers that refer to the dimensions of the entity, along with their typical or default values (Rumelhart, 1980). For example, a schema for snake might include the slots eats, habitat, color, shape, and so forth. A default value for eats might be mice.

According to the model, one interprets a noun-noun combination by filling a slot of the head noun with the modifier noun. Thus, one might interpret robin snake by filling a slot in snake (e.g., the slot eats) with the modifier concept robin to produce the meaning "a snake that eats robins." As a result, the filled slot captures a relation between the objects denoted by the modifier and head concepts. As implied by this example, the concept specialization model assumes that the modifier concept refers to an object or entity (e.g., robin refers to the type of bird that we call robins). However, this approach does not address the interpretations in which the modifier noun is taken to refer to a property, as in the present experiments. These approaches to how concepts combine are not necessarily wrong. Rather, they are incomplete. They do not provide accounts of how a property of the modifier noun is instantiated in the head noun of the combination because they do not address combinations that have these interpretations. My characterization of property instantiation suggests that such models must be augmented with comparison and construction processes.

Induction. The interpretation of a novel noun-noun combination or nominal metaphor is largely a problem of induction. For example, in trying to interpret zebra clam, one must assess which property (or properties) of zebra are likely to apply to clam. Recent approaches to property induction (Heit \& Rubinstein, 1994; Osherson et al., 1990; Sloman, 1993) have focused on modeling these likelihood assessments. However, the present results suggest that properties are not just asserted about a category with some likelihood but rather they are also instantiated. The instantiation process is another aspect of induction because it involves inferences about how a property applies to another concept. So, when interpreting a zebra clam as a clam with stripes, people are not only inferring that a zebra clam has stripes but they are also inferring how those stripes are manifested in zebra clam. Recent approaches to property induction will need to address this aspect of induction.

\section{Conclusion and Summary}

The results of these experiments suggest that the process of combining concepts can involve instantiating a property of one concept in another concept rather than asserting that an abstract property of one concept applies to the second concept. Furthermore, the instantiation process produces a new version of a property that tends to differ from its original source to the extent that the concepts are different. I have described a general processing account of property instantiation that involves two (perhaps interrelated) mechanisms. One mechanism is a comparison and construction process that is influ- 
enced by constraints associated with both the modifier and the head noun concepts. A second mechanism involves extensional feedback, whereby instantiation is achieved through the retrieval of existing referents of the combination. I have also noted that property instantiation is an important aspect of the interpretation of nominal metaphors and the induction of the properties. Models of conceptual combination, induction, metaphor, and analogy need to more seriously address how properties are instantiated.

\section{REFERENCES}

Barsalou, L. W. (1993). Flexibility, structure, and linguistic vagary in concepts: Manifestations of a compositional system of perceptual symbols. In A. C. Collins, S. E. Gathercole, M. A. Conway, \& P. E. M. Morris (Eds.), Theories of memory (pp. 29-101). Hillsdale, NJ: Erlbaum.

Barsalou, L. W., \& HaLe, C. R. (1993). Components of conceptual representation: From feature lists to recursive frames. In I. Van Mechelen, J. Hampton, R. Michalski, \& P. Theuns (Eds.), Categories and concepts: Theoretical views and inductive data analysis ( $\mathrm{pp}$. 97-144). San Diego: Academic Press

COOLEN, R., VAN JAARSVEld, H. J., \& SCHREUder, R. (1991). The interpretation of isolated nominal compounds. Memory \& Cognition, 19, 341-352.

CRuse, D. A. (1986). Lexical semantics. Cambridge: Cambridge University Press.

Downing, P. (1977). On the creation and use of English compound nouns. Language, 53, 810-842.

Falkenhainer, B., Forbus, K. D., \& Gentner, D. (1989). The structure mapping engine: Algorithm and examples. Artificial Intelligence, 41, 1-63.

Finke, R. A. (1989). The principles of mental imagery. Cambridge, MA: MIT Press.

Gagne, C. L., \& Shoben, E. J. (1997). Influence of thematic relations on the comprehension of modifier-noun combinations. Journal of Experimental Psychology: Learning, Memory, \& Cognition, 23, 71-87.

GeNTNER, D. (1983). Structure-mapping: A theoretical framework for analogy. Cognitive Science, 7, 155-170.

GENTNER, D. (1989). The mechanisms of analogical learning. In S. Vosniadou \& A. Ortony (Eds.), Similarity, analogy, and thought (pp. 199241). Cambridge: Cambridge University Press.

Glucksberg, S., Gildea, P., \& BookIn, M. B. (1982). On understanding nonliteral speech: Can people ignore metaphors? Journal of Verbal Learning Behavior, 21, 85-98.

GluCKSBERG, S., \& KeYSAR, B. (1990). Understanding metaphorical comparisons. Beyond similarity. Psychological Review, 97, 3-18.

Glucksberg, S., Manfredi, D., \& MCGlone, M. S. (1997). How metaphors create new categories. In T. B. Ward, S. M. Smith, \& J. Vaid (Eds.), Creative thought: An investigation of conceptual structures and processes (pp. 327-350). Washington, DC: American Psychological Association.

Grice, H. P. (1975). Logic and conversation. In P. Cole \& J. L. Morgan (Eds.), Syntax and semantics: Vol. 3. Speech acts (pp. 41-58). New York: Academic Press.

halff, H. M., Ortony, A., \& Anderson, R. C. (1976). A contextsensitive representation of word meanings. Memory \& Cognition, 4 378-383.

HAMPTON, J. A. (1987). Inheritance of attributes in natural concept conjunctions. Memory \& Cognition, 15, 55-71.

HeIt, E., \& Barsalou, B. (1996). The instantiation principle in natural categories. Memory, 4, 413-451.

Heit, E., \& Rubinstein, J. (1994). Similarity and property effects in inductive reasoning. Journal of Experimental Psychology: Learning, Memory, \& Cognition, 20, 411-422.

Holyoak, K. J., \& Thagard, P. (1989). Analogical mapping by constraint satisfaction. Cognitive Science, 13, 295-355.
Hummel, J. E., \& HolyoAK, K. J. (1997), Distributed representations of structure: A theory of analogical access and mapping. Psychological Review, 104, 427-466.

Lakoff, G., \& JoHnson, M. (1980). Metaphors we live by. Chicago: University of Chicago Press.

Markman, A. B., \& Gentner, D. (1993). Splitting the differences: A structural alignment view of similarity. Journal of Memory \& Language, 32, 517-535.

MARKS, L. E., \& BorNSTEIN, M. H. (1987). Sensory similarities: Classes, characteristics, and cognitive consequences. In R. E. Haskel (Ed.), Cognition and symbolic structures: The psychology of metaphoric transformation (pp. 49-65). Norwood, NJ: Ablex Publishing Corporation.

MARR, D. (1982). Vision. San Francisco: W. H. Freeman.

Medin, D. L., \& Shoben, E. J. (1988). Context and structure in conceptual combination. Cognitive Psychology, 20, 158-190.

MurPhy, G. L. (1988). Comprehending complex concepts. Cognitive Science, 12, 529-562.

Murphy, G. L., \& MediN, D. L. (1985). The role of theories in conceptual coherence. Psychological Review, 92, 289-316.

Ortony, A. (1979). Beyond literal similarity. Psychological Review, 86, $161-180$

Osherson, D. N., Smith, E. E., Wilkie, O., Lopez, A., \& Shafir, E. (1990). Category-based induction. Psychological Review, 97, 185-200.

PALMER, S. E. (1978). Fundamental aspects of cognitive representation. In E. Rosch \& B. B. Lloyd (Eds.), Cognition and categorization (pp. 259-303). Hillsdale, NJ: Erlbaum.

Rips, L. J., \& TurnbulL, W. (1980). How big is big? Relative and absolute properties in memory. Cognition, 8, 145-174.

RuMELHART, D. E. (1980). Schemata: The building blocks of cognition. In R. J. Spiro, B. C. Bruce, \& W. F. Brewer (Eds.), Theoretical issues in reading comprehension (pp. 33-58). Hillsdale, $\mathrm{NJ}$ : Erlbaum.

Sloman, S. A. (1993). Feature-based induction. Cognitive Psychology, 25, 231-280.

SOlOMON, K. O., \& Barsalou, L. W. (in press). Empirical evidence for perceptual simulation in conceptual processing. In C. Taddei-Ferretti \& C. Musio (Eds.), Downward processes in the perception representation mechanisms. London: World Scientific.

Stevens, A., \& Coupe, P. (1978). Distortions in judged spatial relations. Cognitive Psychology, 10, 422-437.

WISNIEWSKI, E. J. (1996). Construal and similarity in conceptual combination. Journal of Memory \& Language, 35, 434-453.

WisNieWski, E. J. (1997). When concepts combine. Psychonomic Bulletin \& Review, 4, 167-183.

WisNieWskı, E. J., \& Love, B. C. (1998). Properties versus relations in conceptual combination. Journal of Memory \& Language, 38, 177 202.

Wisniewski, E. J., \& Markman, A. B. (1993). The role of structural alignment in conceptual combination. In Proceedings of the Fifteenth Annual Conference of the Cognitive Science Society (pp. 1083-1086). Hillsdale, NJ: Erlbaum.

WISNIEWSKI, E. J., \& MEDIN, D. L. (1994). On the interaction of theory and data in concept learning. Cognitive Science, 18, 221-282.

\section{NOTES}

1. All of the item analyses reported in this paper used $t$ tests, pairing similar and dissimilar combinations that shared the same modifier noun.

2. In certain contexts, subjects might prefer the interpretation of a dissimilar combination over a similar combination. For example, suppose that there was a horse with straight, horizontal stripes on its sides. Then, people might prefer the phrase American flag horse over zebra horse to refer to this horse. Even though the instantiation of stripes in zebra horse better matches its instantiation in zebra, the instantiation of stripes in American flag horse better matches the instantiation of stripes in the referent.

3. The omitted pairs were grass carpet/frog carpet and glue paintiglue ribbon. The four combinations that differed from those of Experiment 1 
were rose dandelion, cherry onion, suitcase box, and fish frog, which were used in place of cactus dandelion, radish onion, briefcase box, and fish shark, respectively. Experiment 2 was started before Experiment 1. The items from Experiment 1 that replaced those from Experiment 2 were chosen because they were considered better stimuli. In particular, radish onion and fish shark are more similar combinations than cherry onion and fish frog. It was thought that, for cactus dandelion and briefcase box, the modifier properties "prickly" and "has a handle" were more salient than for rose dandelion and suitcase box.
4. Item analyses were not performed because of the small number of observations per item.

5. Three of the similar combinations and two of the dissimilar combinations had two consensus properties (i.e., ones with the same number of occurrences and at least three occurrences). For two of these similar combinations and one of these dissimilar combinations, one of the consensus properties was the experimenter property.

6 . These models have focused more on analogies than on metaphors, but they assume that both are interpreted in a similar manner.

\section{APPENDIX}

Quadruplets of Combinations and the Properties Associated With Their Interpretations

\begin{tabular}{|c|c|c|}
\hline $\begin{array}{c}\text { Similar } \\
\text { Combination }\end{array}$ & $\begin{array}{l}\text { Dissimilar } \\
\text { Combination }\end{array}$ & Property \\
\hline $\begin{array}{l}\text { yarn rope } \\
\text { pencil stick }\end{array}$ & $\begin{array}{l}\text { pencil rope } \\
\text { yarn stick }\end{array}$ & thin \\
\hline $\begin{array}{l}\text { snow sand } \\
\text { milk soda }\end{array}$ & $\begin{array}{l}\text { milk sand } \\
\text { snow soda }\end{array}$ & white \\
\hline $\begin{array}{l}\text { coffee water } \\
\text { crow robin }\end{array}$ & $\begin{array}{l}\text { crow water } \\
\text { coffee robin }\end{array}$ & black \\
\hline $\begin{array}{l}\text { zebra horse } \\
\text { American flag tablecloth }\end{array}$ & $\begin{array}{l}\text { American flag horse } \\
\text { zebra tablecloth }\end{array}$ & striped \\
\hline $\begin{array}{l}\text { sugar powder } \\
\text { honey syrup }\end{array}$ & $\begin{array}{l}\text { honey powder } \\
\text { sugar syrup }\end{array}$ & sweet \\
\hline $\begin{array}{l}\text { radish onion } \\
\text { firetruck car }\end{array}$ & $\begin{array}{l}\text { firetruck onion } \\
\text { radish car }\end{array}$ & red \\
\hline $\begin{array}{l}\text { apple pear } \\
\text { pearl pebble }\end{array}$ & $\begin{array}{l}\text { pearl pear } \\
\text { apple pebble }\end{array}$ & round \\
\hline $\begin{array}{l}\text { boomerang chopstick } \\
\text { banana cucumber }\end{array}$ & $\begin{array}{l}\text { banana chopstick } \\
\text { boomerang cucumber }\end{array}$ & curved \\
\hline $\begin{array}{l}\text { ostrich duck } \\
\text { giraffe horse }\end{array}$ & $\begin{array}{l}\text { giraffe duck } \\
\text { ostrich horse }\end{array}$ & long neck \\
\hline $\begin{array}{l}\text { skunk squirrel } \\
\text { shit mud }\end{array}$ & $\begin{array}{l}\text { shit squirrel } \\
\text { skunk mud }\end{array}$ & smells bad \\
\hline $\begin{array}{l}\text { whale porpoise } \\
\text { elephant tiger }\end{array}$ & $\begin{array}{l}\text { elephant porpoise } \\
\text { whale tiger }\end{array}$ & large \\
\hline $\begin{array}{l}\text { grass carpet } \\
\text { frog toad }\end{array}$ & $\begin{array}{l}\text { frog carpet } \\
\text { grass toad }\end{array}$ & green \\
\hline $\begin{array}{l}\text { bird mouse } \\
\text { airplane bus }\end{array}$ & $\begin{array}{l}\text { airplane mouse } \\
\text { bird bus }\end{array}$ & can fly \\
\hline $\begin{array}{l}\text { tape ribbon } \\
\text { glue paint }\end{array}$ & $\begin{array}{l}\text { glue ribbon } \\
\text { tape paint }\end{array}$ & sticky \\
\hline $\begin{array}{l}\text { coffee cup bowl } \\
\text { briefcase box }\end{array}$ & $\begin{array}{l}\text { briefcase bowl } \\
\text { coffee cup box }\end{array}$ & with a handle \\
\hline $\begin{array}{l}\text { fish shark } \\
\text { snake lizard }\end{array}$ & $\begin{array}{l}\text { snake shark } \\
\text { fish lizard }\end{array}$ & has scales \\
\hline $\begin{array}{l}\text { scorpion spider } \\
\text { bee fly }\end{array}$ & $\begin{array}{l}\text { bee spider } \\
\text { scorpion fly }\end{array}$ & stings \\
\hline $\begin{array}{l}\text { porcupine pig } \\
\text { cactus dandelion }\end{array}$ & $\begin{array}{l}\text { cactus pig } \\
\text { porcupine dandelion }\end{array}$ & prickly \\
\hline $\begin{array}{l}\text { race car bike } \\
\text { cheetah dog }\end{array}$ & $\begin{array}{l}\text { cheetah bike } \\
\text { race car dog }\end{array}$ & fast \\
\hline
\end{tabular}


APPENDIX (Continued)

\begin{tabular}{lll}
\hline \multicolumn{1}{c}{$\begin{array}{c}\text { Similar } \\
\text { Combination }\end{array}$} & $\begin{array}{l}\text { Dissimilar } \\
\text { Combination }\end{array}$ & Property \\
\hline dice marbles & $\begin{array}{l}\text { Dalmation marbles } \\
\text { dice poodles } \\
\text { Dalmation poodles }\end{array}$ & spots \\
$\begin{array}{l}\text { rooster sparrow } \\
\text { alarm clock television }\end{array}$ & $\begin{array}{l}\text { alock sparrow } \\
\text { rooster television } \\
\text { pencil chalk }\end{array}$ & tent chalk \\
tent roof & pencil roof & pointy \\
\hline
\end{tabular}

(Manuscript received July 3, 1996 ;

revision accepted for publication August 11, 1997.) 